\title{
Glycitin regulates osteoblasts through TGF- $\beta$ or AKT signaling pathways in bone marrow stem cells
}

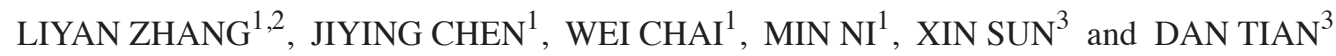 \\ ${ }^{1}$ Department of Orthopedics, General Hospital of Chinese People's Liberation Army, \\ Beijing 100853; ${ }^{2}$ First Department of Orthopedics, Affiliated Hospital of Beihua University; \\ ${ }^{3}$ Life Science Research Center of Beihua University, Jilin 132000, P.R. China
}

Received June 18, 2016; Accepted July 26, 2016

DOI: $10.3892 / \mathrm{etm} .2016 .3696$

\begin{abstract}
The aim of the present study was to examine the effect of glycitin on the regulation of osteoblasts from bone marrow stem cells (BMSCs) through transforming growth factor (TGF)- $\beta$ or protein kinase B (AKT) signaling pathways. BMSCs were extracted from New Zealand white rabbits and used to analyze the effect of glycitin on BMSCs. BMSCs were cleared using xylene and observed via light microscopy. BMSCs were subsequently induced with glycitin $(0.01,0.5,1$, 5 and $10 \mu \mathrm{M}$ ) for 7 days, and stained with Oil Red O. The mechanism of action of glycitin on BMSCs was investigated, in which contact with collagen type I ( $\mathrm{Col} \mathrm{I})$, alkaline phosphatase (ALP), TGF- $\beta$ and AKT was studied. Firstly, BMSCs appeared homogeneously mazarine blue, and which showed that BMSCs were successful extracted. Administration of glycitin increased cell proliferation and promoted osteoblast formation from BMSCs. Furthermore, glycitin activated the gene expression of Col I and ALP in BMSCs. Notably, glycitin suppressed protein expression of TGF- $\beta$ and AKT in BMSCs. These results indicated that glycitin may regulate osteoblasts through TGF- $\beta$ or AKT signaling pathways in BMSCs.
\end{abstract}

\section{Introduction}

Osteanagenesis, which is also known as bone tissue engineering, is a novel and multidisciplinary field (1). By employing the fundamental principles of biology, medical science and tissue engineering, it is possible to remodel injured bone tissue or cure bone diseases (2). Resulting from trauma or physiological

Correspondence to: Mr. Jiying Chen, Department of Orthopedics, General Hospital of Chinese People's Liberation Army, 28 Fuxing Road, Beijing 100853, P.R. China

E-mail: chenjiying_301@yeah.net

Mrs. Xin Sun, Life Science Research Center of Beihua University, 3999 Binjiangdong Road, Jilin 132000, P.R. China

E-mail:sunxinbh@126.com

Key words: glycitin, osteoblast, bone marrow stem cells, transforming growth factor- $\beta 1$, protein kinase B and pathological bone resorption, bone defects are a global heath issue, the treatment of which can be challenging. Among craniofacial and plastic surgeries, bone defects caused by trauma are a common clinical problem, and osteanagenesis may be beneficial in repairing injuries and improving the quality of life of patients (3). Osteanagenesis is also used to treat and repair bone mass in various bone diseases caused by gender, age and infection, including osteoporosis, osteopenia and tooth loss as a result of periodontitis (4).

Bone formation is a lengthy and strictly regulated process associated with embryonic development, reconstruction of bone tissue and bone fracture repair (5). Classical bone biology theories claim that mature osteoblasts are formed from BMSCs. During bone formation, bone precursor cells are differentiated into mature osteoblasts that can compound and secrete bone matrix (6), and are subsequently mineralized and imbedded into bone matrix. During the embryonic development process, BMSCs participate in bone formation through membranous ossification and entochondrostosis (7). Membranous ossification predominantly occurs in craniofacial bones, parts of the cartilage and mandibles. During the developmental process of these tissues, BMSCs are able to directly differentiate into osteoblasts, whereas entochondrostosis is employed in the development process of torso and limb bones (8). Initially, through accumulation, proliferation and differentiation into chondrocytes, BMSCs begin to form bones (9). Cartilage cells are then gradually divided into hypertrophic chondrocytes. With the mineralization of deep stromas and in-growth of blood vessels, chondrocytes die and are replaced by osteoblasts, and mature bones are formed.

As a bioactivator, soy isoflavone is a type of flavonoid compound and a secondary metabolite formed during the growth of soybeans (10). Although it is extracted from plants, it has the same structure as estrogen; therefore, it is also known as phytoestrogen. Soy isoflavone can occur in various types, including daidzin, daidzein, genistin, genistein, glycitin and glycitein (11). Glycitin is antibacterial, antiviral and estrogenic (10), and has been demonstrated to have preventative effects on alcoholism, cardiovascular and cerebrovascular diseases and some types of cancer $(12,13)$. It can also reduce the number of tumors and alleviate or avoid macteric syndrome caused by a decrease of estrogen (14), and has demonstrated anti-aging effects (15). The aim of the present study was to 
investigate whether glycitin regulates osteoblast formation from BMSCs through TGF- $\beta$ or AKT signaling pathways.

\section{Materials and methods}

Isolation and culture of primary BMSCs. A total of 24 healthy male New Zealand white rabbits (age, 6 months; weight, $1-1.5 \mathrm{~kg}$ ) were purchased from the Experimental Animal Center of Jilin City (Jilin, China). Rabbits were housed at $22-24^{\circ} \mathrm{C}$ (relative humidity, 55-70\%) with natural light and air circulation, and were allowed free access to food and water. All animal procedures were conducted in strict accordance with the Animal Ethical Standard, and the present study was approved by the Experimental Animal Center of Beihua University, Jilin Province Ethics Committee (Jilin, China).

BMSCs were isolated from New Zealand white rabbits using the method described previously by Li et al (14). Initially, the femurs and tibias were removed, and flushed bone marrow cells were acquired via Percoll density gradient centrifugation $(1.073 \mathrm{~g} / \mathrm{ml})$. Flushed bone marrow cells were washed with phosphate-buffered saline (PBS) and seeded into $25-\mathrm{cm}^{2}$ cell culture flasks. Flushed bone marrow cells were incubated with L-Dulbecco's modified Eagle medium (DMEM) supplemented with $10 \%$ fetal bovine serum and $1 \%$ penicillin-streptomycin at $37^{\circ} \mathrm{C}$ in an atmosphere containing $5 \% \mathrm{CO}_{2}$ for $48 \mathrm{~h}$, and subsequently incubated with DMEM for $48 \mathrm{~h}$. Cells were detached using $0.25 \%$ trypsin and $0.02 \%$ EDTA (Merck Millipore, Darmstadt, Germany) and centrifuged at 2,000 $\mathrm{x} g$ for $5 \mathrm{~min}$. Suspended cells were gathered, seeded on 6-well plates at $1.5-2 \times 10^{6}$ cells/well and incubated for two days.

Authenticating BMSCs. BMSCs were fixed using 5\% precooled paraformaldehyde for $30 \mathrm{~min}$ at $4^{\circ} \mathrm{C}$ and incubated with hematoxylin (Merck Millipore) for $10 \mathrm{~min}$. BMSCs were washed with water for $10 \mathrm{~min}$, and 95\% ethyl alcohol and xylene were used to dehydrate and clear BMSCs, respectively. BMSCs were observed using light microscopy (D5300; Nikon Corp., Tokyo, Japan).

Assessment of primary BMSCs growth. BMSCs $\left(1-2 \times 10^{6}\right.$ cells or $1-2 \times 10^{4}$ per well) were cultured in 6 - or 96 -well culture plates overnight at $37^{\circ} \mathrm{C}$ in an atmosphere containing $5 \% \mathrm{CO}_{2}$. Glycitin (Merck Millipore) was added to the wells at final concentrations of $0.01,0.5,1,5$ and $10 \mu \mathrm{M}$ and cultured for 7 days.

In cells cultured in 6-well culture plates, BMSCs were determined using Oil Red O staining and observed via light microscopy at $510 \mathrm{~nm}$. BMSCs were fixed using 5\% precooled paraformaldehyde for $30 \mathrm{~min}$ at $4^{\circ} \mathrm{C}$ and stained with $0.6 \%$ (w/v) Oil Red O solution for $15 \mathrm{~min}$ at room temperature. Cells stained with Oil Red $\mathrm{O}$ were washed with water $(3 \times 5 \mathrm{~min})$ to remove unbound dye, and culture dishes were stained with $1 \mathrm{ml}$ isopropyl alcohol for $10 \mathrm{~min}$.

In cells cultured in 96-well culture plates, BMSCs were determined via MTT assay. A total of $20 \mu \mathrm{l}$ MTT (5 g/l) were added to each well and cultured for $4 \mathrm{~h}$. The supernatant was removed and $200 \mu$ l dimethylsulfoxide were added to each well for $15 \mathrm{~min}$. Optical density (OD) was measured using a microplate spectrophotometer (model 680; Bio-Rad Laboratories,
Inc., Hercules, CA, USA) at $570 \mathrm{~nm}$. Proliferation rate was calculated using: OD treated / OD control x 100\%.

Measurement of collagen type 1 (Col I) and alkaline phosphatase $(A L P)$ using reverse-transcription polymerase chain reaction (RT-PCR). Total RNA was extracted from BMSCs treated with glycitin $(0,0.5,1$ and $5 \mu \mathrm{M})$ using TRIzol reagent (Invitrogen; Thermo Fisher Scientific, Inc., Waltham, MA, USA). Total RNA (1-2 $\mu \mathrm{g})$ was used to transcribe cDNA using a SYBR PrimeScript RT-PCR kit (Takara Bio, Inc., Otsu, Japan), according to the manufacturer's protocol PCR was performed on an ABI 7500 Real-Time PCR system (Applied Biosystems; Thermo Fisher Scientific, Inc.). PCR thermal cycling was performed as follows: Amplification at $94^{\circ} \mathrm{C}$ for $1 \mathrm{~min}$, followed by 40 cycles of amplification at $94^{\circ} \mathrm{C}$ for $30 \mathrm{sec}$, annealing at $58^{\circ} \mathrm{C}$ for $45 \mathrm{sec}$, and extension at $72^{\circ} \mathrm{C}$ for $30 \mathrm{sec}$. Primers were designed as follows: Col I, forward 5'-TGACCTCAAGATGTGCCACT-3' and reverse 5'-GGG AGTTTCCATGAAGCCAC-3'; and $\beta$-actin forward 5'-CGT GCGGGACATCAAGGA-3' and reverse 5'-AGGAAGGAG GGCTGGAACA-3'. Subsequently, 7500 Fast Real-Time PCR system software was used to analyze crossing threshold (Cq) values using the second derivative maximum method (16).

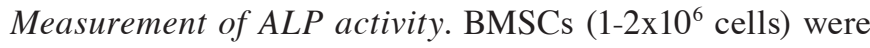
cultured in 6-well plates overnight at $37^{\circ} \mathrm{C}$ in an atmosphere containing $5 \% \mathrm{CO}_{2}$. Glycitin was added to the wells at final concentrations of $0,0.5,1$ and $5 \mu \mathrm{M}$ and cultured for 7 days. Cells were washed with ice-cold PBS and lysed via the repeated freeze-thaw method. Supernatant was analyzed using an ALP kit (Sangon Biotech Co., Ltd., Shanghai, China). ALP activity was calculated according to the formula: Treated group / control x $100 \%$.

Western blotting for TGF- $\beta$ and phosphorylated AKT ( $p$-AKT). Proteins were extracted from BMSCs treated with glycitin $(0,0.5,1$ and $5 \mu \mathrm{M})$ by grinding with protease inhibitors. BMSCs were lysed using RIPA lysis buffer and protein content was measured using a micro-Bradford assay kit (Sangon Biotech Co., Ltd.). Protein samples (50-100 $\mu \mathrm{g})$ were separated using 10-12\% SDS-PAGE and transferred onto a polyvinylidene fluoride membrane. Membranes were incubated with 5\% non-fat milk for $2 \mathrm{~h}$ at room temperature, and were subsequently incubated with antibodies against TGF- $\beta(1: 500$; sc-146), p-AKT $(1: 500 ;$ sc-135,650) and $\beta$-actin $(1: 1,000$; sc-130,656; all Santa Cruz Biotechnology, Inc., Dallas, TX, USA) at $4^{\circ} \mathrm{C}$ for $24 \mathrm{~h}$. Membranes were washed three times with Tris-buffered saline with Tween-20 and subsequently incubated with an anti-rabbit secondary antibody (1:5,000; C2247; Applygen Technologies Inc., Beijing, China) at $37^{\circ} \mathrm{C}$ for $30 \mathrm{~min}$ at room temperature and were visualized with enhanced chemiluminescent reagent (ECL Plus; P0018A; Beyotime Institute of Biotechnology, Haimen, China). Proteins were quantified using Image Lab 4.1 (Bio-Rad Laboratories, Inc.).

Statistical analysis. Data are presented as the mean \pm standard deviation using SPSS software (version 18.0; SPSS, Inc., Chicago, IL, USA). Statistical differences were analyzed using Student's t-test. $\mathrm{P}<0.05$ was considered to indicate a statistically significant difference. 


\section{Results}

Authentication of BMSCs. The constitutional formula of glycitin is displayed in Fig. 1. BMSCs appeared homogeneously mazarine blue, which demonstrated that BMSCs were successful extracted, indicating that BMSCs were successfully separated and cultivated (Fig. 2).

Glycitin promotes BMSC proliferation. MTT assay was used to measure the effect of glycitin on the proliferation of BMSCs. As shown in Fig. 3, glycitin increased the proliferation of BMSCs, with statistical significance detected after treatment with 1 and $5 \mu \mathrm{M}$ glycitin $(\mathrm{P}=0.0023$ and $\mathrm{P}=0.0004$, respectively).

Glycitin increases adipogenic differentiation of BMSCs. As shown in Fig. 4, Oil Red $\mathrm{O}$ staining was observed in every group. A marked increase in red staining, and therefore osteoblasts, was detected following treatment with 1 and $5 \mu \mathrm{M}$ glycitin.

Glycitin increases Col I mRNA expression and ALP activity in BMSCs. In order to elucidate the effect of glycitin on the mRNA expression levels and activity of Col I and ALP, respectively, RT-PCR and ALP kits were used. The results demonstrated that administration of 1 and $5 \mu \mathrm{M}$ glycitin significantly promoted Col I mRNA expression ( $\mathrm{P}=0.0079$ and $\mathrm{P}=0.0031$, respectively) and ALP activity in BMSCs $(\mathrm{P}=0.0049$ and $\mathrm{P}=0.0023$, respectively; Fig. 5).

Glycitin increases TGF- $\beta$ expression levels in BMSCs. To confirm the effect of glycitin on TGF- $\beta$ signaling in BMSCs, TGF- $\beta$ protein expression of BMSCs was detected. The results indicated that pretreatment with 1 and $5 \mu \mathrm{M}$ glycitin significantly enhanced TGF- $\beta$ protein expression of BMSCs ( $\mathrm{P}=0.0063$ and $\mathrm{P}=0.0021$, respectively; Fig. 6).

Glycitin increases $p$-AKT levels in BMSCs. To investigate the effect of glycitin on p-AKT in BMSCs, p-AKT was measured using western blotting. The results demonstrated that 1 and $5 \mu \mathrm{M}$ glycitin significantly increased the presence of $\mathrm{p}$-AKT in BMSCs $(\mathrm{P}=0.0071$ and $\mathrm{P}=0.0033$, respectively; Fig. 7).

\section{Discussion}

Old bones and osteoblasts are absorbed by osteoclasts and new bones are formed (17). During continuous reconstruction, the equilibrium of the re-constructional process is regulated by a complicated signal network consisting of hormones, growth factors, cytokines, chemo-tactic factors and mechanical signals (18). BMSCs are capable of osteogenic differentiation, which indicates the prospect of the clinical application for biotherapy based on BMSCs (19). Osteogenic differentiation of BMSCs, in vitro and in vivo, has been extensively studied $(20,21)$. The results of the present study demonstrated that glycitin promotes cell proliferation, osteoblast induction, and activates Col I mRNA expression and ALP activity of BMSCs. Li et al (14) reported that daidzin, genistin, and glycitin affects osteogenic and adipogenic differentiation.

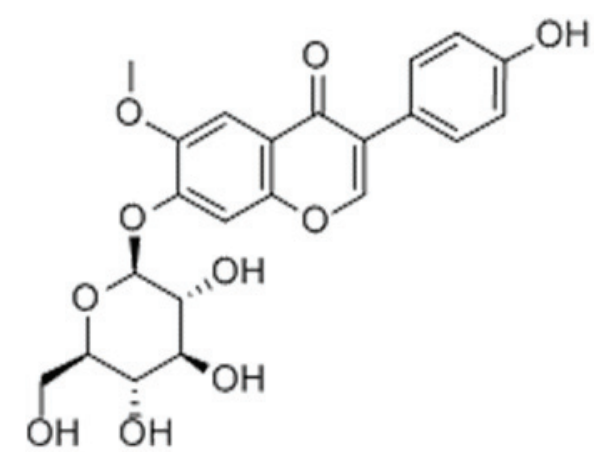

Figure 1. Constitutional formula of glycitin.
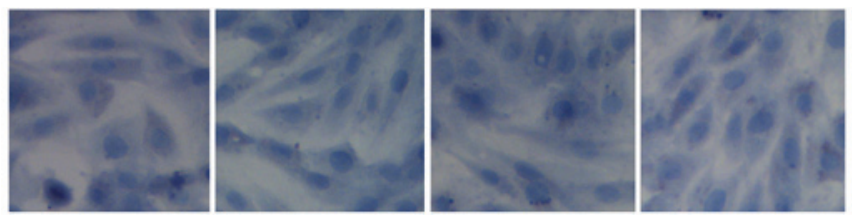

Figure 2. Authentication of bone marrow stem cells, as detected by hematoxylin (magnification, $\mathrm{x} 400$ ).

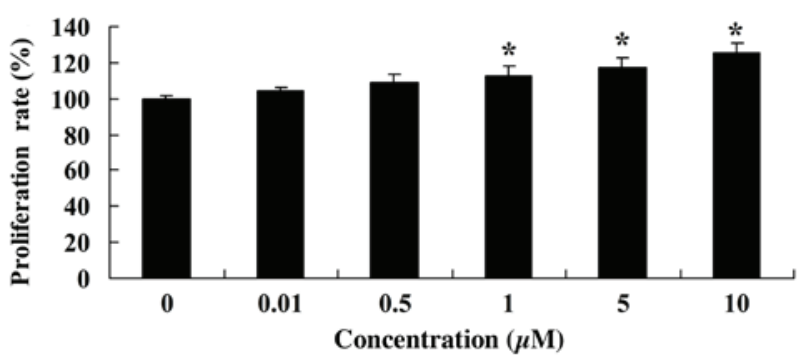

Figure 3. Glycitin promotes the proliferation of bone marrow stem cells ${ }^{*} \mathrm{P}<0.01$ vs. the $0 \mu \mathrm{M}$ glycitin-treated group.
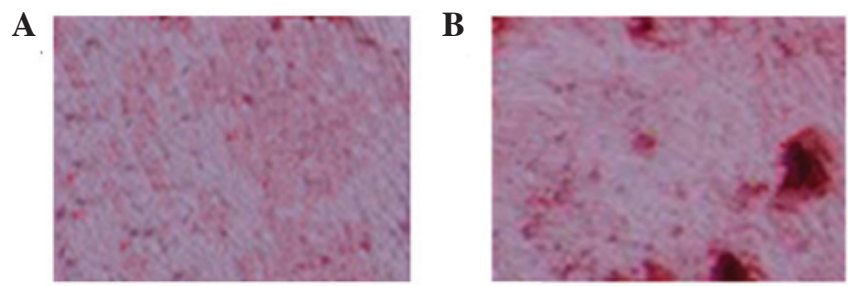

C

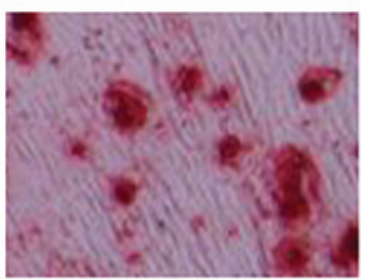

$\mathbf{D}$

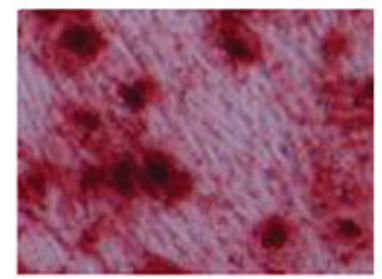

Figure 4. Adipogenic differentiation of bone marrow stem cells, as detected by Oil Red O staining (magnification, $\mathrm{x} 400$ ). (A) 0 , (B) 0.5 , (C) 1 and (D) $5 \mu \mathrm{M}$ glycitin-treated BMSCs.

The TGF- $\beta$ protein super family includes TGF, activins, inhibin and bone morphogenetic proteins (22). These proteins have important roles in cell proliferation, differentiation, formation of cell matrix, the formation of tissues and organs, 


\section{A}

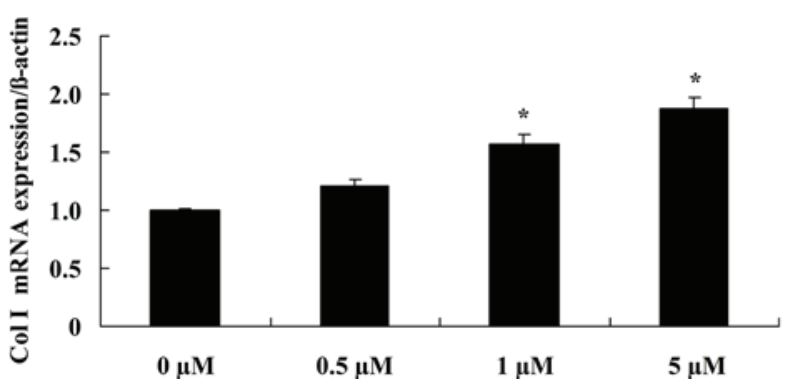

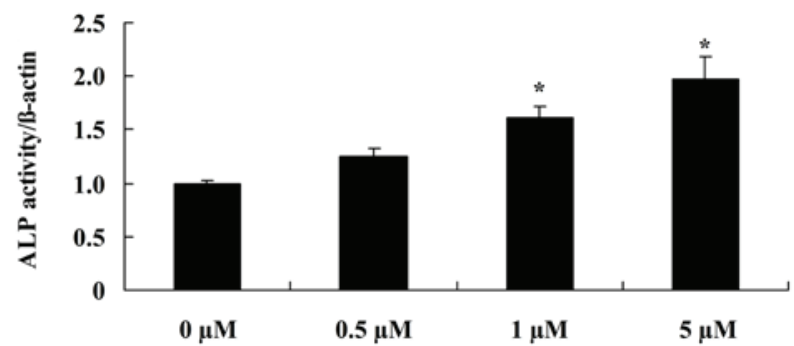

Figure 5. Effect of glycitin on Col I and ALP mRNA expression levels in BMSCs. (A) Col I and (B) ALP mRNA expression levels were analyzed in BMSCs after treatment with glycitin. ${ }^{*} \mathrm{P}<0.01$ vs. the $0 \mu \mathrm{M}$ glycitin-treated group. Col I, collagen type I; ALP, alkaline phosphatase; BMSCs, bone marrow stem cells.

A

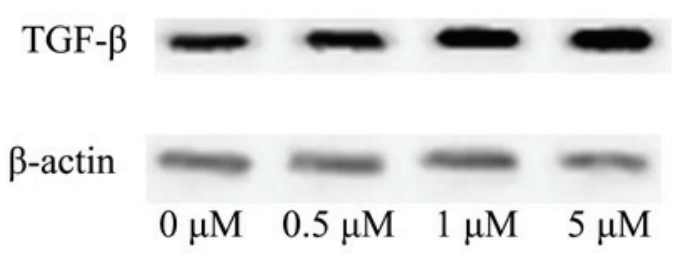

B

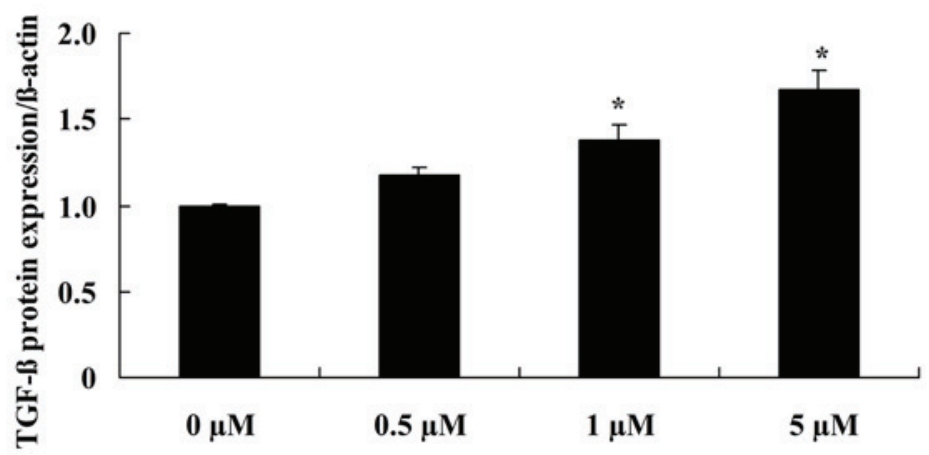

Figure 6. Effect of glycitin on TGF- $\beta$ signaling of BMSCs. The effect of glycitin on TGF- $\beta$ protein expression levels was assessed by (A) western blotting assays and (B) subsequent statistical analysis of TGF- $\beta$ protein expression levels in BMSCs. "P $<0.01$ vs. the $0 \mu$ M glycitin-treated group. TGF- $\beta$, transforming growth factor beta-1; BMSCs, bone marrow stem cells.

A

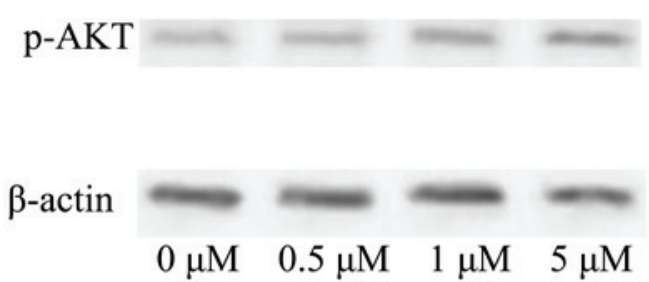

B

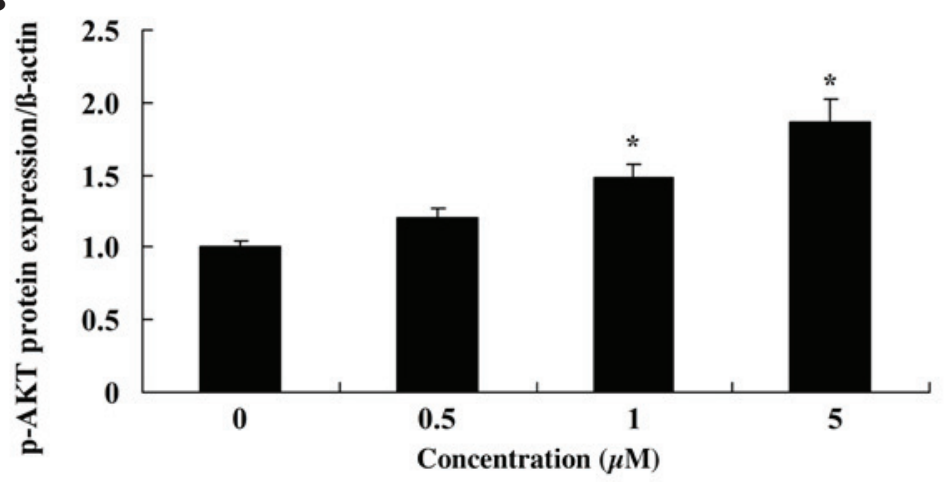

Figure 7. Effect of glycitin on p-AKT of BMSCs. The effect of glycitin on p-AKT protein expression levels was assessed by (A) western blotting assays and (B) subsequent statistical analysis of p-AKT protein expression levels in BMSCs. " $\mathrm{P}<0.01$ vs. the $0 \mu \mathrm{M}$ glycitin-treated group. P-AKT, phosphorylated AKT; BMSCs, bone marrow stem cells.

embryonic development and immunoregulation. During bone formation, the combination of TGF- $\beta$ and its ligand may inhibit the formation of osteoclasts and bone absorption, but may additionally facilitate the osteogenesis of osteoblasts (23). Recent research has shown that TGF- $\beta 1$ may promote the osteogenic differentiation of hMSCs and induce the expression of osteogenesis genes, ALP, collagen type I and osteocalcins (24). Conversely, the addition of TGF- $\beta 3$ may inhibit the expression of ALP, suggesting that the TGF- $\beta$ signaling pathway has a different role in the osteogenic differentiation of hMSCs (25). In addition, it was demonstrated that glycitin significantly enhanced T GF- $\beta$ protein expression levels in BMSCs. Kim et al (26) indicated that glycitin promotes the proliferation and migration of human dermal fibroblast cells through TGF- $\beta$ signaling.

Through the above data analysis, it was indicated that oxygen deficit and Ang II may induce the phosphorylation of Akt, a downstream modifier of PI3K, which can alter downstream modifiers and lead to changes in cell proliferation (27). Glycitin significantly advanced p-AKT formation in BMSCs. 
Kim et al (26) indicated that glycitin promotes proliferation and migration of human dermal fibroblast cells through TGF- $\beta$ and p-AKT signaling.

In conclusion, the results of the present study demonstrated that glycitin promotes cell proliferation and induces osteoblast differentiation in BMSCs. A notable finding was that molecules endowed with activating Col I mRNA expression, ALP activity, and TGF- $\beta$ and p-AKT signaling participated in the effect of glycitin regulating osteoblasts in BMSCs.

\section{Acknowledgements}

This research was supported by the National Natural Science Foundation of China (grant no. 81301,564) and the Army Medical Science Youth Training Project (grant no. 13QNP184), the 'Twelfth Five-year' science and technology research project of Jilin Department of Education (grant no. 2015-141) and the Jilin province Department of Project (grant no. 20130624003JC).

\section{References}

1. Mathews S, Bhonde R, Gupta PK and Totey S: Extracellular matrix protein mediated regulation of the osteoblast differentiation of bone marrow derived human mesenchymal stem cells. Differentiation 84: 185-192, 2012.

2. Yamachika E, Tsujigiwa H, Matsubara M, Hirata Y, Kita K, Takabatake K, Mizukawa N, Kaneda Y, Nagatsuka H and Iida S: Basic fibroblast growth factor supports expansion of mouse compact bone-derived mesenchymal stem cells (MSCs) and regeneration of bone from MSC in vivo. J Mol Histol 43: 223-233, 2012.

3. Staudt ND, Aicher WK, Kalbacher H, Stevanovic S, Carmona AK, Bogyo M and Klein G: Cathepsin X is secreted by human osteoblasts, digests CXCL-12 and impairs adhesion of hematopoietic stem and progenitor cells to osteoblasts. Haematologica 95: 1452-1460, 2010.

4. Yang C, Liu Y, Li C and Zhang B: Repair of mandibular defects by bone marrow stromal cells expressing the basic fibroblast growth factor transgene combined with multi-pore mineralized Bio-Oss. Mol Med Rep 7: 99-104, 2013.

5. Kim BS, Kang HJ, Park JY and Lee J: Fucoidan promotes osteoblast differentiation via JNK- and ERK-dependent BMP2-Smad 1/5/8 signaling in human mesenchymal stem cells. Exp Mol Med 47: e128, 2015.

6. Glynn ER, Londono AS, Zinn SA, Hoagland TA and Govoni KE: Culture conditions for equine bone marrow mesenchymal stem cells and expression of key transcription factors during their differentiation into osteoblasts. J Anim Sci Biotechnol 4: 40, 2013

7. Lee HW, Kim SY, Kim AY, Lee EJ, Choi JY and Kim JB: Adiponectin stimulates osteoblast differentiation through induction of COX2 in mesenchymal progenitor cells. Stem Cells 27: 2254-2262, 2009.

8. Clabaut A, Delplace S, Chauveau C, Hardouin P and Broux O: Human osteoblasts derived from mesenchymal stem cells express adipogenic markers upon coculture with bone marrow adipocytes. Differentiation 80: 40-45, 2010.

9. Barbier V, Winkler IG, Wadley R and Lévesque JP: Flow cytometry measurement of bone marrow perfusion in the mouse and sorting of progenitors and stems cells according to position relative to blood flow in vivo. Methods Mol Biol 844: 45-63, 2012.

10. Zhang YB, Chen WH, Guo JJ, Fu ZH, Yi C, Zhang M and Na XL: Soy isoflavone supplementation could reduce body weight and improve glucose metabolism in non-Asian postmenopausal women-a meta-analysis. Nutrition 29: 8-14, 2013.
11. Xu Z, Wu Q and Godber JS: Stabilities of daidzin, glycitin, genistin, and generation of derivatives during heating. J Agric Food Chem 50: 7402-7406, 2002.

12. Robb EL and Stuart JA: Multiple phytoestrogens inhibit cell growth and confer cytoprotection by inducing manganese superoxide dismutase expression. Phytother Res 28: 120-131, 2014.

13. Hsu A, Bray TM, Helferich WG, Doerge DR and Ho E: Differential effects of whole soy extract and soy isoflavones on apoptosis in prostate cancer cells. Exp Biol Med (Maywood) 235: 90-97, 2010.

14. Li XH, Zhang JC, Sui SF and Yang MS: Effect of daidzin, genistin, and glycitin on osteogenic and adipogenic differentiation of bone marrow stromal cells and adipocytic transdifferentiation of osteoblasts. Acta Pharmacol Sin 26: 1081-1086, 2005.

15. Zang Y, Igarashi K and Yu C: Anti-obese and anti-diabetic effects of a mixture of daidzin and glycitin on C57BL/6J mice fed with a high-fat diet. Biosci Biotechnol Biochem 79: $117-123,2015$.

16. Li W, Ling W, Teng X, Quan C, Cai S and Hu S: Effect of advanced glycation end products, extracellular matrix metalloproteinase inducer and matrix metalloproteinases on type-I collagen metabolism. Biomed Rep 4: 691-693, 2016.

17. Peng KY, Horng LY, Sung HC, Huang HC and Wu RT: Antiosteoporotic Activity of Dioscorea alata L. cv. Phyto through driving mesenchymal stem cells differentiation for bone formation. Evid Based Complement Alternat Med 2011: $712892,2011$.

18. Han DS, Chang HK, Kim KR and Woo SM: Consideration of bone regeneration effect of stem cells: Comparison of bone regeneration between bone marrow stem cells and adipose-derived stem cells. J Craniofac Surg 25: 196-201, 2014.

19. Mosig RA and Martignetti JA: Loss of MMP-2 in murine osteoblasts upregulates osteopontin and bone sialoprotein expression in a circuit regulating bone homeostasis. Dis Model Mech 6: 397-403, 2013.

20. Gao M, Chen J, Lin G, Li S, Wang L, Qin A, Zhao Z, Ren L, Wang Y and Tang BZ: Long-Term tracking of the osteogenic differentiation of mouse BMSCs by aggregation-induced emission nanoparticles. ACS Appl Mater Interfaces 8: 17878-17884, 2016.

21. Wang C, Liu D, Zhang C, Sun J, Feng W, Liang XJ, Wang S and Zhang J: Defect-Related luminescent hydroxyapatite-enhanced osteogenic differentiation of bone mesenchymal stem cells via an ATP-induced cAMP/PKA pathway. ACS Appl Mater Interfaces 8: 11262-11271, 2016.

22. Batlle R, Alba-Castellón L, Loubat-Casanovas J, Armenteros E, Francí C, Stanisavljevic J, Banderas R, Martin-Caballero J, Bonilla F, Baulida J, et al: Snaill controls TGF- $\beta$ responsiveness and differentiation of mesenchymal stem cells. Oncogene 32: 3381-3389, 2013.

23. Kumar A, Ruan M, Clifton K, Syed F, Khosla S and Oursler MJ: TGF- $\beta$ mediates suppression of adipogenesis by estradiol through connective tissue growth factor induction. Endocrinology 153: 254-263, 2012.

24. Claros S, Rico-Llanos GA, Becerra J and Andrades JA: A novel human TGF- $\beta 1$ fusion protein in combination with rhBMP-2 increases chondro-osteogenic differentiation of bone marrow mesenchymal stem cells. Int J Mol Sci 15: 11255-11274, 2014.

25. Yin XX, Chen ZQ, Liu ZJ, Ma QJ and Dang GT: Icariine stimulates proliferation and differentiation of human osteoblasts by increasing production of bone morphogenetic protein 2 . Chin Med J (Engl) 120: 204-210, 2007.

26. Kim YM, Huh JS, Lim Y and Cho M: Soy isoflavone glycitin (4'-Hydroxy-6-Methoxyisoflavone-7-D-Glucoside) promotes human dermal fibroblast cell proliferation and migration via TGF- $\beta$ signaling. Phytother Res 29: 757-769, 2015.

27. Wang C, Lin K, Chang J and Sun J: Osteogenesis and angiogenesis induced by porous $\beta-\mathrm{CaSiO}$ (3)/PDLGA composite scaffold via activation of AMPK/ERK1/2 and PI3K/Akt pathways. Biomaterials 34: 64-77, 2013. 